\title{
Very Long Chain Fatty Acid
}

National Cancer Institute

\section{Source}

National Cancer Institute. Very Long Chain Fatty Acid. NCI Thesaurus. Code C68439.

A class of dietary fatty acids that have an alkyl chain leng th above 21 carbon atoms. 